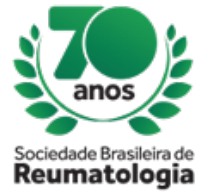

\title{
A CASE OF TENDINOUS XANTHOMA REVEALED BY ULTRASONOGRAPHIC EXAMINATION
}

Claudia Valeria Vierhout (Hospital PUC Campinas, Campinas, SP, Brasil), Nadia Regina Bossolan Schincariol (Hospital PUC Campinas, Campinas, SP, Brasil), Jose Alexandre Mendonça (Hospital PUC Campinas, Campinas, SP, Brasil), Lucas Eduardo Pedri (Hospital PUC Campinas, Campinas, SP, Brasil), Andre Marun Lyrio (Hospital PUC Campinas, Campinas, SP, Brasil), Rubens Bonfiglioli (Hospital PUC Campinas, Campinas, SP, Brasil), Jose Roberto Provenza (Hospital PUC Campinas, Campinas, SP, Brasil), Vanessa Ramos Guissa (Hospital PUC Campinas, Campinas, SP, Brasil), Fernanda Bertucci Sanches (Hospital PUC Campinas, Campinas, SP, Brasil), Thais Campos Ferreira Pinto (Hospital PUC Campinas, Campinas, SP, Brasil), Igor Tadeu Garcia Ferreira (Hospital PUC Campinas, Campinas, SP, Brasil)

\section{BACKGROUND}

Tendinous xanthomas are cholesterol deposits in tendons. They can occur in places like Achilles tendons, extensor tendons of the hand and elbow. When it comes on Achilles tendons, it occurs preferentially in women, between 30-40 years of age. It is commonly associated with familial hypercholesterolemia $(\mathrm{FH})$, an autosomal dominant inherited disorder, characterized by high LDL cholesterol levels and premature coronary disease. Symptoms may vary according to the magnitude of the lesions: small lesions are usually asymptomatic. Larger lesions can manifest with pain, skin irritation or even deforming masses. Ultrasonography (US) and Magnetic Resonance Imaging (MRI) are the great allies in diagnosis, being able to detect the disease even in the pre-clinical phases. The following case report describes how US helped in the diagnosis of this rare disorder.

\section{CASE REPORT}

A 26-year-old female patient with mechanical pain in the region of insertion of the Achilles tendon bilaterally. She had performed treatment with non-steroidal anti-inflammatory drugs (NSAIDs) and physical therapy, without significant pain improvement. She has hyperlipidemia since the age of 24 , but without adequate treatment. Physical examination revealed bilateral thickening of the Achilles tendon and presence of xanthelasms located in the eyelids. Laboratory tests (including ESR and CRP) were normal, except for an increase in total cholesterol. Ultrasound examination detected significant thickening of the Achilles tendon bilaterally, with diffuse heterogeneous pattern - findings compatible with tendon xanthoma. Initiated statin for patient and referred to orthopaedist for evaluation of removal of xanthomas.

\section{CONCLUSION}

The hypothesis of tendinous xanthoma should always be reminded when patient presents clinical and laboratorial signs of hypercolesterolemia associated with tendinopathies. The early diagnosis is fundamental, since the beginning of treatment can change the course of the disease and prevent coronaryopathy. Ultrasonography is a simple, fast and accurate tool to define the diagnosis. 